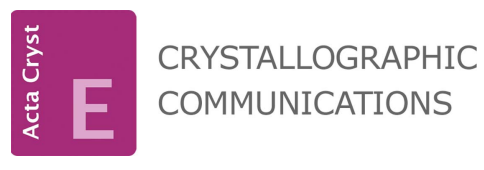

ISSN 2056-9890

Received 4 April 2018

Accepted 9 April 2018

Edited by D.-J. Xu, Zhejiang University (Yuquan Campus), China

Keywords: crystal structure; natural product; phenanthrene; $\mathrm{C}-\mathrm{H}$. . O hydrogen bond; $\mathrm{C}-$ $\mathrm{H} \cdots \pi$ interactions.

CCDC reference: 1835842

Supporting information: this article has supporting information at journals.iucr.org/e

\section{Crystal structure of $(4 \mathrm{bS}, 8 \mathrm{a} R)$-1-isopropyl-4b,8,8- trimethyl-7-oxo-4b,7,8,8a,9,10-hexahydro- phenanthren-2-yl acetate}

\author{
Yassine Laamari, ${ }^{a}$ Moulay Youssef Ait Itto, ${ }^{a}$ Abdelkhalek Riahi, ${ }^{\text {b }}$ Sylviane \\ Chevreux, $^{b}$ Aziz Auhmani ${ }^{*}$ and El Mostafa Ketatnic
}

\begin{abstract}
aLaboratory of Organic Synthesis and Physico-Molecular Chemistry, Department of Chemistry, Faculty of Sciences Semlalia, BP 2390, Marrakech 40001, Morocco, ${ }^{\mathbf{b}}$ Institute of Molecular Chemistry of Reims, CNRS UMR 7312 Bat. Europol'Agro, Moulin of the Housse UFR Sciences, BP 1039-51687 Reims Cedex 2, France, and 'Laboratory of Applied Spectro-Chemistry and Environment, University Sultan Moulay Slimane, Faculty of Science and Technology, PO Box 523 , Beni-Mellal, Morocco. *Correspondence e-mail: a.auhmani@uca.ac.ma
\end{abstract}

The title compound, $\mathrm{C}_{22} \mathrm{H}_{28} \mathrm{O}_{3}$, was prepared by a direct acetylation reaction of naturally occurring totarolenone. The molecule contains three fused rings, which exhibit different conformations. The central ring has a half-chair conformation, while the non-aromatic oxo-substituted ring has a screw-boat conformation. In the crystal, molecules are linked by $\mathrm{C}-\mathrm{H} \cdots \mathrm{O}$ hydrogen bonds and $\mathrm{C}-\mathrm{H} \cdots \pi$ interactions, forming sheets parallel to the $b c$ plane. The carbonyl $\mathrm{O}$ atoms and the $\mathrm{C}$ atom at the 6-position of the cyclohexene ring are each disordered over two sets of sites with major occupancy components of 0.63 (7) and 0.793 (14), respectively.

\section{Chemical context}

Diterpene phenols are a family of natural products isolated from a variety of terrestrial plant sources. They exhibit a wide variety of interesting biological activities such as antitumour (Iwamoto et al., 2003; Son et al. 2005), antimicrobial (Yoshikawa et al., 2008; Pereda-Miranda et al., 1992), antiviral (Yang et al., 2011; Wen et al., 2007) and anti-inflammatory (Chen et al. 2013). In addition, derivatives of diterpene phenol natural products have been studied extensively as potential chemotherapeutic agents (Areche et al., 2007; Yang et al. 2001).

With the aim of preparing diterpene phenol derivatives, we report here the hemisynthesis (Fig. 1) of $(4 \mathrm{~b} S, 8 \mathrm{a} R)-1$ isopropyl-4 b,8,8-trimethyl-7-oxo-4 b,7,8,8a,9,10-hexahydrophenanthren-2-yl acetate, 2 , from naturally occurring totarolenone, 1, extracted from the heartwood of Tetraclinis articulata (Chow et al., 1960). Treatment of $\mathbf{1}$ with acetic anhydride and pyridine provides compound $\mathbf{2}$ as colourless crystals in $88 \%$ yield. X-ray single crystal structure analysis allowed its structure to be confirmed unambiguously. Its<smiles>[R20][R4](=O)C(C)(C)OC(C)=O</smiles>

Figure 1

Reaction scheme for the synthesis of the title compound 2 . 
structure was also characterized by ${ }^{1} \mathrm{H}$ and ${ }^{13} \mathrm{C}$ NMR measurements.<smiles>CC(=O)Oc1ccc2c(c1C(C)C)CC[C@@H]1C(C)(C)C(=O)C=C[C@@]21C</smiles>

\section{Structural commentary}

The molecular structure is built up from three fused sixmembered rings (Fig. 2). In the molecule, there are two chiral carbon atoms, C4b exhibits an $S$ configuration and C8a exhibits an $R$ configuration. The central six-membered ring ( $\mathrm{C} 4 A$, $\mathrm{C} 4 B, \mathrm{C} 8 A, \mathrm{C} 9, \mathrm{C} 10, \mathrm{C} 10 A$ ) assumes a half-chair conformation, as indicated by the total puckering amplitude $Q_{\mathrm{T}}=0.55$ (2) $\AA$ and spherical polar angle $\theta=51.0$ (2) ${ }^{\circ}$ with $\varphi=136.0(2)^{\circ}$. The major component of the cyclohexene ring exhibits a screwboat conformation $\left[Q_{\mathrm{T}}=0.462(2) \AA, \theta=113.7(2)^{\circ}, \varphi=\right.$ $\left.145.9(2)^{\circ}\right]$ while the minor component has a chair conformation $\left[Q_{\mathrm{T}}=0.558(6) \AA, \theta=159.4(6)^{\circ}, \varphi=235.6(14)^{\circ}\right]$.

\section{Supramolecular features}

In the crystal, molecules are linked by $\mathrm{C}-\mathrm{H} \cdots \mathrm{O}$ and $\mathrm{C}-$ $\mathrm{H} \cdots \pi$ interactions, forming layers parallel to the $b c$ plane (Table 1 and Fig. 3).

\section{Database survey}

A search of the Cambridge Structural Database using the $1,2,3,4,4 \mathrm{a}, 9,10,10 \mathrm{a}-\mathrm{h}$ xahydrophenanthren ring system (Fig. $4 a$ ) as the main skeleton, revealed the presence of 75 structures. These include several compounds similar to the title compound. One with a similar conformation has a hydroxyl substituent in place of the acetate in the title

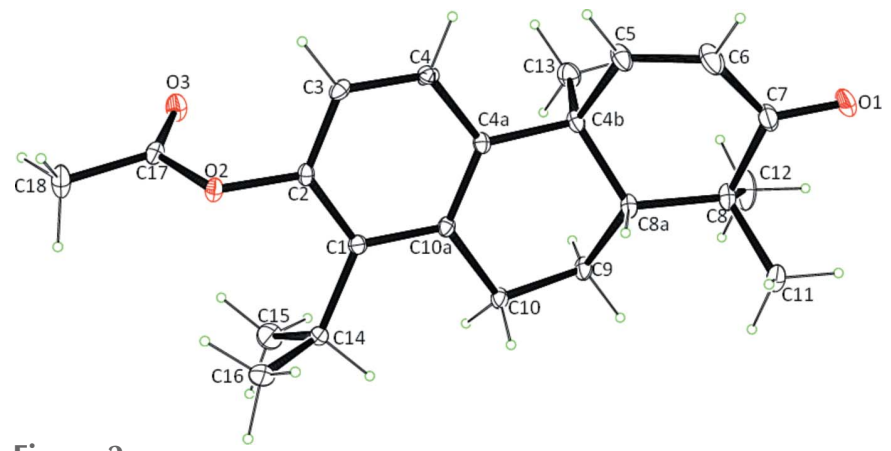

Figure 2

The molecular structure of the title compound with the atom-numbering scheme. The displacement ellipsoids are drawn at the $30 \%$ probability level. Only the major disorder components are shown.
Table 1

Hydrogen-bond geometry $\left(\AA{ }^{\circ}\right)$.

$C g 1$ is the centroid of the benzene ring.

\begin{tabular}{lllll}
\hline$D-\mathrm{H} \cdots A$ & $D-\mathrm{H}$ & $\mathrm{H} \cdots A$ & $D \cdots A$ & $D-\mathrm{H} \cdots A$ \\
\hline $\mathrm{C} 4-\mathrm{H} 4 \cdots \mathrm{O} 1^{\mathrm{i}}$ & 0.95 & 2.50 & $3.411(10)$ & 159 \\
$\mathrm{C} 4-\mathrm{H} 4 \cdots \mathrm{O} 1 A^{\mathrm{i}}$ & 0.95 & 2.55 & $3.428(17)$ & 154 \\
$\mathrm{C} 10-\mathrm{H} 10 B \cdots \mathrm{O} 1 A^{\mathrm{ii}}$ & 0.99 & 2.55 & $3.328(17)$ & 136 \\
$\mathrm{C} 13-\mathrm{H} 13 C \cdots \mathrm{O} 3^{\text {iii }}$ & 0.98 & 2.59 & $3.530(2)$ & 161 \\
$\mathrm{C} 18-\mathrm{H} 18 A \cdots C g 1^{\text {iv }}$ & 0.98 & 2.55 & $3.504(2)$ & 165 \\
\hline
\end{tabular}

Symmetry codes: (i) $-x+1, y-\frac{1}{2},-z$; (ii) $-x, y-\frac{1}{2},-z$; (iii) $-x+1, y+\frac{1}{2},-z+1$; (iv) $-x+1, y-\frac{1}{2},-z+1$.

compound (Benharref et al., 2011), and three others have a methoxy group in position $4 \mathrm{~b}$ and carbaldehyde/benzenesulfonohydrazide (Vo et al., 2008) or biphenylsulfonyl (Gu \& You, 2011) in position 9 (Fig. 4b), while six entries (Oubabi et al., 2014a,b; Zeroual et al., 2007, 2008; Cutfield et al., 1974; Pettit et al., 2004) have 1-isopropyl-4b,8,8-trimethyl substituents (Fig. 4c).

\section{Synthesis and crystallization}

A solution of totarolenone $\mathbf{1}(300 \mathrm{mg}, 1.041 \mathrm{mmol})$ in acetic anhydride $(10 \mathrm{~mL})$ and sodium acetate $(290 \mathrm{mg})$ was heated under reflux for $24 \mathrm{~h}$. After cooling, the solution was extracted with ether $(3 \times 20 \mathrm{~mL})$. The organic layer was washed with water, dried on anhydrous $\mathrm{Na}_{2} \mathrm{SO}_{4}$ and evaporated under reduced pressure. The obtained residue was chromatographed on silica gel column using hexane and ethyl acetate (95/5) as eluent, to give compound 2.

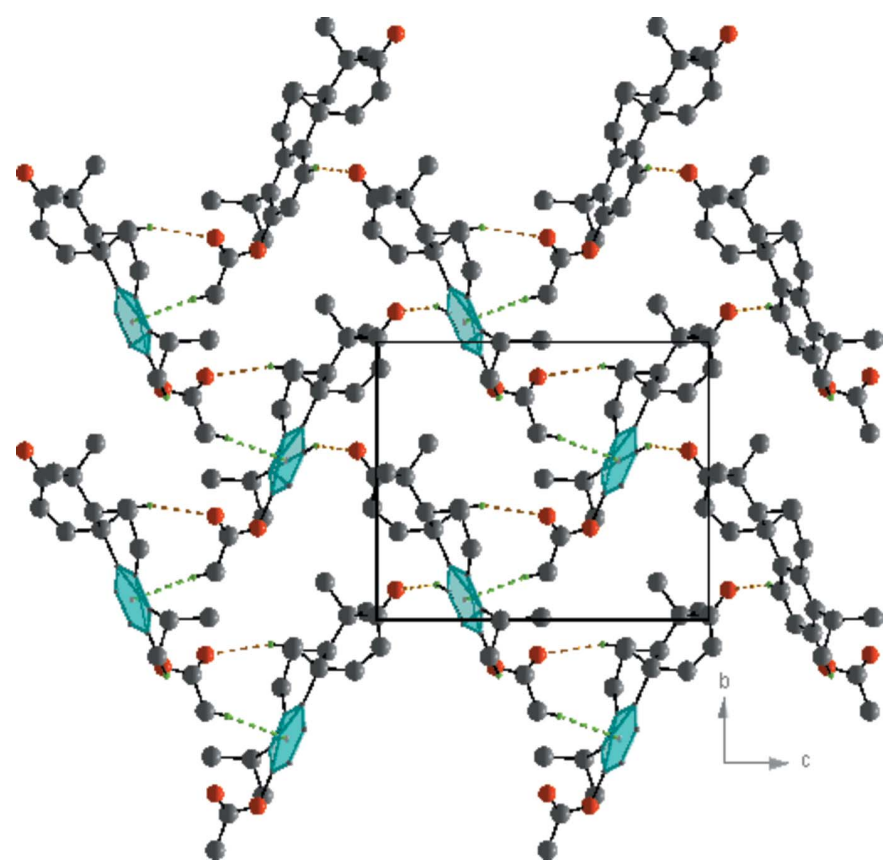

Figure 3

A view along the $a$ axis of the crystal packing of the title compound, showing the $\mathrm{C}-\mathrm{H} \cdots \mathrm{O}$ hydrogen bonds (orange dashed lines) and $\mathrm{C}-$ $\mathrm{H} \cdots \pi$ interactions (green dashed lines). For clarity, only the $\mathrm{H}$ atoms involved in these interactions have been included. 
<smiles>c1ccc2c(c1)CCC1CCCCC21</smiles>

(a)<smiles>CC(C)c1cccc2c1CC[C@@H]1C(C)(C)CCC[C@]21C</smiles>

(c)<smiles>O=C1C=CC2c3ccccc3CCC2C1</smiles><smiles>CC(=O)Oc1ccc2c(c1C(C)C)CC[C@@H]1C(C)(C)C(=O)C=C[C@@]21C</smiles>

title compound
Figure 4

The core structures for database survey: (a) 1,2,3,4,4a,9,10,10a-hexahydrophenanthren, and its $(b)$ 7-oxo with double bond between $\mathrm{C} 5$ and C6, (c) 1-isopropyl-4 b,8,8-trimethyl substituents; and $(d)$ the title compound.

\section{Refinement}

Crystal data, data collection and structure refinement details are summarized in Table $2 . \mathrm{H}$ atoms were placed in calculated positions and refined in the riding model: $\mathrm{C}-\mathrm{H}=0.95-1.00 \AA$ with $U_{\text {iso }}(\mathrm{H})=1.2 U_{\text {eq }}(\mathrm{C})$ or $1.5 U_{\text {eq }}(\mathrm{C}$-methyl $)$. The carbonyl O atom is disordered over two sites having occupancies of 0.63 (7) and 0.37 (7). Atom C6 atom of the cyclohexene ring is disordered over two sites with an occupancy ratio of 0.793 (14):0.207 (14). The absolute structure was reliably determined based on the value of the Flack parameter [0.02 (5)].

\section{Funding information}

Funding for this research was provided by: Cadi Ayyad University.

\section{References}

Areche, C., Rodríguez, J. A., Razmilic, I., Yáñez, T., Theoduloz, C. \& Schmeda-Hirschmann, G. (2007). J. Pharm. Pharmacol. 59, 289300.

Benharref, A., Lassaba, E., Mazoir, N., Daran, J.-C. \& Berraho, M. (2011). Acta Cryst. E67, o906.

Brandenburg, K. \& Putz, H. (2012). DIAMOND. Crystal Impact GbR, Bonn, Germany.

Bruker (2012). APEX2, SAINT and SADABS. Bruker AXS Inc., Madison, Wisconsin, USA.

Chen, Y.-C., Li, Y.-C., You, B.-J., Chang, W.-T., Chao, L.-K., Lo, L.-C., Wang, S.-Y., Huang, G.-J. \& Kuo, Y.-H. (2013). Molecules, 18, 682689.

Chow, Y. L. \& Erdtman, H. (1960). Acta Chem. Scand. 14, 1852-1853.

Cutfield, F., Waters, T. N. \& Clark, G. R. (1974). J. Chem. Soc. Perkin Trans. 2, pp. 150-157.

Farrugia, L. J. (2012). J. Appl. Cryst. 45, 849-854.

Gu, Q. \& You, S.-L. (2011). Org. Lett. 13, 5192-5195.

Iwamoto, M., Minami, T., Tokuda, H., Ohtsu, H. \& Tanaka, R. (2003). Planta Med. 69, 69-72.

Oubabi, R., Auhmani, A., Ait Itto, M. Y., Auhmani, A. \& Daran, J.-C. (2014a). Acta Cryst. E70, o317.

Oubabi, R., Auhmani, A., Ait Itto, M. Y., Auhmani, A. \& Daran, J.-C. (2014b). Acta Cryst. E70, o866-0867.
Table 2

Experimental details.

\begin{tabular}{|c|c|}
\hline \multicolumn{2}{|l|}{ Crystal data } \\
\hline Chemical formula & $\mathrm{C}_{22} \mathrm{H}_{28} \mathrm{O}_{3}$ \\
\hline$M_{\mathrm{r}}$ & 340.44 \\
\hline Crystal system, space group & Monoclinic, $P 2_{1}$ \\
\hline Temperature $(\mathrm{K})$ & 100 \\
\hline$a, b, c(\AA)$ & $7.4103(2), 10.4681(3), 12.8121(3)$ \\
\hline$\beta\left({ }^{\circ}\right)$ & $102.235(1)$ \\
\hline$V\left(\AA^{6}\right)$ & $971.28(4)$ \\
\hline$Z$ & 2 \\
\hline Radiation type & $\mathrm{Cu} K \alpha$ \\
\hline$\mu\left(\mathrm{mm}^{-1}\right)$ & 0.60 \\
\hline Crystal size $(\mathrm{mm})$ & $0.41 \times 0.30 \times 0.18$ \\
\hline \multicolumn{2}{|l|}{ Data collection } \\
\hline Diffractometer & D8 Venture CMOS area detector \\
\hline Absorption correction & $\begin{array}{l}\text { Numerical (SADABS; Bruker, } \\
\text { 2012) }\end{array}$ \\
\hline $\begin{array}{l}\text { No. of measured, independent and } \\
\text { observed }[I>2 \sigma(I)] \text { reflections }\end{array}$ & $17946,3909,3852$ \\
\hline$R_{\text {int }}$ & 0.029 \\
\hline$(\sin \theta / \lambda)_{\max }\left(\AA^{-1}\right)$ & 0.625 \\
\hline \multicolumn{2}{|l|}{ Refinement } \\
\hline$R\left[F^{2}>2 \sigma\left(F^{2}\right)\right], w R\left(F^{2}\right), S$ & $0.030,0.077,1.05$ \\
\hline No. of reflections & 3909 \\
\hline No. of parameters & 252 \\
\hline No. of restraints & 1 \\
\hline $\mathrm{H}$-atom treatment & $\mathrm{H}$-atom parameters constrained \\
\hline$\Delta \rho_{\max }, \Delta \rho_{\min }\left(\mathrm{e} \AA^{-3}\right)$ & $0.16,-0.17$ \\
\hline Absolute structure & $\begin{array}{l}\text { Flack } x \text { determined using } 1757 \\
\quad \text { quotients }\left[\left(I^{+}\right)-\left(I^{-}\right)\right] /\left[\left(I^{+}\right)+\left(I^{-}\right)\right] \\
\quad \text { (Parsons } \text { et al., } 2013)\end{array}$ \\
\hline Absolute structure parameter & $0.02(5)$ \\
\hline
\end{tabular}

Computer programs: APEX2 and SAINT (Bruker, 2012), SHELXS2014 (Sheldrick, 2008), SHELXL2014 (Sheldrick, 2015), ORTEP-3 for Windows (Farrugia, 2012), DIAMOND (Brandenburg et al., 2012), PLATON (Spek, 2009) and publCIF (Westrip, 2010).

Parsons, S., Flack, H. D. \& Wagner, T. (2013). Acta Cryst. B69, 249259.

Pereda-Miranda, R., Hernández, L. \& López, R. (1992). Planta Med. 58, 223-224.

Pettit, G. R., Tan, R., Northen, J. S., Herald, D. L., Chapuis, J. C. \& Pettit, R. K. (2004). J. Nat. Prod. 67, 1476-1482.

Sheldrick, G. M. (2008). Acta Cryst. A64, 112-122.

Sheldrick, G. M. (2015). Acta Cryst. C71, 3-8.

Son, K.-H., Oh, H.-M., Choi, S.-K., Han, D. C. \& Kwon, B.-M. (2005). Bioorg. Med. Chem. Lett. 15, 2019-2021.

Spek, A. L. (2009). Acta Cryst. D65, 148-155.

Vo, N. T., Pace, R. D. M., O'Har, F. \& Gaunt, M. J. (2008). J. Am. Chem. Soc. 130, 404-405.

Wen, C.-C., Kuo, Y.-H., Jan, J.-T., Liang, P.-H., Wang, S.-Y., Liu, H.-G., Lee, C.-K., Chang, S.-T., Kuo, C.-J., Lee, S.-S., Hou, C.-C., Hsiao, P.-W., Chien, S.-C., Shyur, L.-F. \& Yang, N.-S. (2007). J. Med. Chem. 50, 4087-4095.

Westrip, S. P. (2010). J. Appl. Cryst. 43, 920-925.

Yang, Z., Kitano, Y., Chiba, K., Shibata, N., Kurokawa, H., Doi, Y., Arakawa, Y. \& Tada, M. (2001). Bioorg. Med. Chem. 9, 347-356.

Yang, L.-B., Li, L., Huang, S.-X., Pu, H.-X., Zhao, Y., Ma, Y.-B., Chen, J.-J., Leng, C.-H., Tao, Z.-M. \& Sun, H.-D. (2011). Chem. Pharm. Bull. 59, 1102-1105.

Yoshikawa, K., Kokudo, N., Tanaka, M., Nakano, T., Shibata, H., Aragaki, N., Higuchi, T. \& Hashimoto, T. (2008). Chem. Pharm. Bull. 56, 89-92.

Zeroual, A., Mazoir, N., Berraho, M., Auhmani, A. \& Benharref, A. (2007). Acta Cryst. E63, o3497-03498.

Zeroual, A., Mazoir, N., Daran, J.-C., Akssira, M. \& Benharref, A. (2008). Acta Cryst. E64, o604-0605. 


\section{supporting information}

Acta Cryst. (2018). E74, 728-730 [https://doi.org/10.1107/S2056989018005510]

Crystal structure of $(4 \mathrm{bS}, 8 \mathrm{a} R)$-1-isopropyl-4b,8,8-trimethyl-7-

oxo-4b,7,8,8a,9,10-hexahydrophenanthren-2-yl acetate

Yassine Laamari, Moulay Youssef Ait Itto, Abdelkhalek Riahi, Sylviane Chevreux, Aziz Auhmani and El Mostafa Ketatni

Computing details

Data collection: APEX2 (Bruker, 2012); cell refinement: SAINT (Bruker, 2012); data reduction: SAINT (Bruker, 2012); program(s) used to solve structure: SHELXS2014 (Sheldrick, 2008); program(s) used to refine structure: SHELXL2014 (Sheldrick, 2015); molecular graphics: ORTEP-3 for Windows (Farrugia, 2012) and DIAMOND (Brandenburg et al., 2012); software used to prepare material for publication: PLATON (Spek, 2009) and publCIF (Westrip, 2010).

(4bS,8aR)-1-Isopropyl-4b,8,8-trimethyl-7-oxo-4b,7,8,8a,9,10-hexahydrophenanthren-2-yl acetate

Crystal data

$\mathrm{C}_{22} \mathrm{H}_{28} \mathrm{O}_{3}$

$M_{r}=340.44$

Monoclinic, $P 2$

$a=7.4103(2) \AA$

$b=10.4681(3) \AA$

$c=12.8121(3) \AA$

$\beta=102.235(1)^{\circ}$

$V=971.28(4) \AA^{3}$

$Z=2$

\section{Data collection}

D8 Venture CMOS area detector diffractometer

Radiation source: microsource $\varphi$ and $\omega$ scans

Absorption correction: numerical

(SADABS; Bruker, 2012)

17946 measured reflections

\section{Refinement}

Refinement on $F^{2}$

Least-squares matrix: full

$R\left[F^{2}>2 \sigma\left(F^{2}\right)\right]=0.030$

$w R\left(F^{2}\right)=0.077$

$S=1.05$

3909 reflections

252 parameters

1 restraint
$F(000)=368$

$D_{\mathrm{x}}=1.164 \mathrm{Mg} \mathrm{m}^{-3}$

$\mathrm{Cu} K \alpha$ radiation, $\lambda=1.54178 \AA$

Cell parameters from 3909 reflections

$\theta=3.5-74.5^{\circ}$

$\mu=0.60 \mathrm{~mm}^{-1}$

$T=100 \mathrm{~K}$

Block, colourless

$0.41 \times 0.30 \times 0.18 \mathrm{~mm}$

3909 independent reflections

3852 reflections with $I>2 \sigma(I)$

$R_{\text {int }}=0.029$

$\theta_{\text {max }}=74.5^{\circ}, \theta_{\min }=3.5^{\circ}$

$h=-9 \rightarrow 9$

$k=-13 \rightarrow 13$

$l=-16 \rightarrow 15$

Hydrogen site location: inferred from neighbouring sites

$\mathrm{H}$-atom parameters constrained

$w=1 /\left[\sigma^{2}\left(F_{\mathrm{o}}^{2}\right)+(0.0376 P)^{2}+0.2106 P\right]$

where $P=\left(F_{\mathrm{o}}{ }^{2}+2 F_{\mathrm{c}}{ }^{2}\right) / 3$

$(\Delta / \sigma)_{\max }<0.001$

$\Delta \rho_{\max }=0.16 \mathrm{e} \AA^{-3}$

$\Delta \rho_{\min }=-0.17$ e $\AA^{-3}$ 
Absolute structure: Flack $x$ determined using

1757 quotients $\left[\left(I^{+}\right)-\left(I^{-}\right)\right] /\left[\left(I^{+}\right)+\left(I^{-}\right)\right]$(Parsons et al., 2013)

Absolute structure parameter: 0.02 (5)

\section{Special details}

Geometry. All esds (except the esd in the dihedral angle between two 1.s. planes) are estimated using the full covariance matrix. The cell esds are taken into account individually in the estimation of esds in distances, angles and torsion angles; correlations between esds in cell parameters are only used when they are defined by crystal symmetry. An approximate (isotropic) treatment of cell esds is used for estimating esds involving l.s. planes.

Fractional atomic coordinates and isotropic or equivalent isotropic displacement parameters $\left(\AA^{2}\right)$

\begin{tabular}{|c|c|c|c|c|c|}
\hline & $x$ & $y$ & $z$ & $U_{\text {iso }} * / U_{\text {eq }}$ & Occ. $(<1)$ \\
\hline $\mathrm{O} 2$ & $0.31864(16)$ & -0.17015 & $0.35741(9)$ & $0.0169(2)$ & \\
\hline $\mathrm{O} 3$ & $0.57128(17)$ & $-0.12685(12)$ & $0.48549(10)$ & 0.0228 & \\
\hline $\mathrm{C} 1$ & $0.1762(2)$ & $0.03631(15)$ & $0.31947(13)$ & $0.0149(3)$ & \\
\hline $\mathrm{C} 2$ & $0.3194(2)$ & $-0.04717(15)$ & $0.31317(12)$ & $0.0141(3)$ & \\
\hline $\mathrm{C} 3$ & $0.4591(2)$ & $-0.01616(16)$ & $0.26178(12)$ & 0.0165 & \\
\hline H3 & 0.5532 & -0.0764 & 0.2575 & $0.020^{*}$ & \\
\hline $\mathrm{C} 4$ & $0.4606(2)$ & $0.10335(17)$ & $0.21673(12)$ & 0.0160 & \\
\hline $\mathrm{H} 4$ & 0.5571 & 0.1253 & 0.1817 & $0.019^{*}$ & \\
\hline $\mathrm{C} 4 \mathrm{~A}$ & $0.3228(2)$ & $0.19262(15)$ & $0.22171(12)$ & $0.0140(3)$ & \\
\hline $\mathrm{C} 4 \mathrm{~B}$ & $0.3352(2)$ & $0.32561(16)$ & $0.17177(13)$ & $0.0167(3)$ & \\
\hline $\mathrm{C} 5$ & $0.3964(3)$ & $0.31576(18)$ & $0.06569(14)$ & $0.0242(4)$ & \\
\hline H5 & 0.4747 & 0.2476 & 0.0544 & $0.029^{*}$ & \\
\hline $\mathrm{C} 7$ & $0.2283(3)$ & $0.5140(2)$ & $-0.00216(14)$ & $0.0262(4)$ & \\
\hline $\mathrm{C} 8$ & $0.1175(3)$ & $0.51452(17)$ & $0.08559(13)$ & $0.0217(4)$ & \\
\hline $\mathrm{C} 8 \mathrm{~A}$ & $0.1410(2)$ & $0.38675(17)$ & $0.14900(12)$ & 0.0166 & \\
\hline $\mathrm{H} 8 \mathrm{~A}$ & 0.0595 & 0.3243 & 0.1021 & $0.020^{*}$ & \\
\hline C9 & $0.0693(2)$ & $0.39088(17)$ & $0.25247(13)$ & $0.0195(3)$ & \\
\hline H9A & 0.1618 & 0.4325 & 0.3095 & $0.023^{*}$ & \\
\hline H9B & -0.0461 & 0.4415 & 0.2413 & $0.023^{*}$ & \\
\hline $\mathrm{C} 10$ & $0.0326(2)$ & $0.25550(16)$ & $0.28606(13)$ & 0.0181 & \\
\hline H10A & 0.0204 & 0.2569 & 0.3615 & $0.022 *$ & \\
\hline H10B & -0.0866 & 0.2260 & 0.2423 & $0.022 *$ & \\
\hline $\mathrm{C} 10 \mathrm{~A}$ & $0.1817(2)$ & $0.16011(16)$ & $0.27480(12)$ & 0.0143 & \\
\hline C11 & $-0.0867(3)$ & $0.5279(2)$ & $0.02894(17)$ & 0.0308 & \\
\hline H11A & -0.1038 & 0.6076 & -0.0122 & $0.046^{*}$ & \\
\hline H11B & -0.1639 & 0.5294 & 0.0823 & $0.046^{*}$ & \\
\hline $\mathrm{H} 11 \mathrm{C}$ & -0.1223 & 0.4554 & -0.0194 & $0.046^{*}$ & \\
\hline $\mathrm{C} 12$ & $0.1723(4)$ & $0.63433(19)$ & $0.15482(19)$ & $0.0409(6)$ & \\
\hline $\mathrm{H} 12 \mathrm{~A}$ & 0.1463 & 0.7108 & 0.1101 & $0.061^{*}$ & \\
\hline H12B & 0.3044 & 0.6310 & 0.1874 & $0.061^{*}$ & \\
\hline $\mathrm{H} 12 \mathrm{C}$ & 0.1011 & 0.6374 & 0.2111 & $0.061^{*}$ & \\
\hline $\mathrm{C} 13$ & $0.4868(3)$ & 0.40095 (19) & $0.24898(16)$ & $0.0252(4)$ & \\
\hline H13A & 0.5014 & 0.4854 & 0.2188 & $0.038^{*}$ & \\
\hline H13B & 0.6039 & 0.3543 & 0.2592 & $0.038^{*}$ & \\
\hline $\mathrm{H} 13 \mathrm{C}$ & 0.4511 & 0.4109 & 0.3180 & $0.038^{*}$ & \\
\hline
\end{tabular}




\begin{tabular}{|c|c|c|c|c|c|}
\hline C14 & $0.0212(2)$ & $-0.00178(18)$ & 0.37495 (14) & $0.0220(4)$ & \\
\hline H14 & -0.0789 & 0.0631 & 0.3538 & $0.026^{*}$ & \\
\hline $\mathrm{C} 15$ & $0.0844(3)$ & $0.0071(3)$ & $0.49646(16)$ & $0.0347(5)$ & \\
\hline $\mathrm{H} 15 \mathrm{~A}$ & 0.1850 & -0.0538 & 0.5209 & $0.052 *$ & \\
\hline H15B & -0.0193 & -0.0132 & 0.5300 & $0.052 *$ & \\
\hline $\mathrm{H} 15 \mathrm{C}$ & 0.1280 & 0.0939 & 0.5163 & $0.052 *$ & \\
\hline $\mathrm{C} 16$ & $-0.0662(3)$ & $-0.1325(2)$ & $0.3410(2)$ & $0.0352(5)$ & \\
\hline H16A & -0.0992 & -0.1373 & 0.2629 & $0.053^{*}$ & \\
\hline H16B & -0.1774 & -0.1433 & 0.3701 & $0.053^{*}$ & \\
\hline $\mathrm{H} 16 \mathrm{C}$ & 0.0224 & -0.2003 & 0.3685 & $0.053^{*}$ & \\
\hline $\mathrm{C} 17$ & $0.4501(2)$ & $-0.19878(16)$ & $0.44565(13)$ & $0.0165(3)$ & \\
\hline $\mathrm{C} 18$ & $0.4186(3)$ & $-0.32928(18)$ & $0.48463(16)$ & $0.0285(4)$ & \\
\hline $\mathrm{H} 18 \mathrm{~A}$ & 0.5095 & -0.3469 & 0.5506 & $0.043^{*}$ & \\
\hline H18B & 0.4316 & -0.3924 & 0.4302 & $0.043 *$ & \\
\hline $\mathrm{H} 18 \mathrm{C}$ & 0.2939 & -0.3344 & 0.4988 & $0.043 *$ & \\
\hline C6 & $0.3407(7)$ & $0.4028(4)$ & $-0.0124(3)$ & $0.0322(13)$ & $0.793(14)$ \\
\hline H6 & 0.3774 & 0.3905 & -0.0784 & $0.039 *$ & $0.793(14)$ \\
\hline C6A & $0.4227(16)$ & $0.4506(11)$ & $0.0182(8)$ & $0.014(3)$ & $0.207(14)$ \\
\hline H6A & 0.5335 & 0.4861 & 0.0047 & $0.017 *$ & $0.207(14)$ \\
\hline $\mathrm{O} 1$ & $0.222(3)$ & $0.6074(7)$ & $-0.0588(15)$ & $0.040(3)$ & $0.63(7)$ \\
\hline O1A & $0.185(3)$ & $0.575(6)$ & -0.084 & $0.059(7)$ & $0.37(7)$ \\
\hline
\end{tabular}

Atomic displacement parameters $\left(\AA^{2}\right)$

\begin{tabular}{lllllll}
\hline & $U^{11}$ & $U^{22}$ & $U^{33}$ & $U^{12}$ & $U^{13}$ & $U^{23}$ \\
\hline O2 & $0.0188(5)$ & $0.0125(5)$ & $0.0173(5)$ & $-0.0001(4)$ & $-0.0009(4)$ & $0.0017(4)$ \\
O3 & $0.0257(6)$ & $0.0197(6)$ & $0.0195(6)$ & $-0.0038(5)$ & $-0.0032(5)$ & $0.0020(5)$ \\
C1 & $0.0147(7)$ & $0.0179(8)$ & $0.0122(7)$ & $0.0008(6)$ & $0.0026(6)$ & $0.0017(6)$ \\
C2 & $0.0173(8)$ & $0.0117(7)$ & $0.0116(7)$ & $-0.0003(6)$ & $-0.0006(6)$ & $0.0008(6)$ \\
C3 & $0.0167(7)$ & $0.0189(8)$ & $0.0139(7)$ & $0.0058(6)$ & $0.0028(6)$ & $-0.0016(6)$ \\
C4 & $0.0153(8)$ & $0.0205(8)$ & $0.0131(7)$ & $0.0004(6)$ & $0.0050(6)$ & $-0.0002(6)$ \\
C4A & $0.0182(8)$ & $0.0139(7)$ & $0.0095(7)$ & $0.0002(6)$ & $0.0022(6)$ & $0.0000(6)$ \\
C4B & $0.0233(8)$ & $0.0145(7)$ & $0.0135(7)$ & $-0.0003(6)$ & $0.0067(6)$ & $0.0014(6)$ \\
C5 & $0.0375(10)$ & $0.0191(8)$ & $0.0201(8)$ & $0.0019(8)$ & $0.0153(7)$ & $0.0021(7)$ \\
C7 & $0.0286(9)$ & $0.0265(9)$ & $0.0229(8)$ & $-0.0030(8)$ & $0.0040(7)$ & $0.0099(8)$ \\
C8 & $0.0302(9)$ & $0.0153(8)$ & $0.0193(8)$ & $0.0023(7)$ & $0.0043(7)$ & $0.0048(7)$ \\
C8A & $0.0248(8)$ & $0.0129(7)$ & $0.0124(7)$ & $0.0018(6)$ & $0.0043(6)$ & $0.0017(6)$ \\
C9 & $0.0277(9)$ & $0.0150(7)$ & $0.0178(8)$ & $0.0080(7)$ & $0.0095(6)$ & $0.0019(6)$ \\
C10 & $0.0223(8)$ & $0.0177(8)$ & $0.0166(8)$ & $0.0066(6)$ & $0.0093(6)$ & $0.0047(6)$ \\
C10A & $0.0173(7)$ & $0.0152(7)$ & $0.0105(7)$ & $0.0031(6)$ & $0.0030(6)$ & $0.0009(5)$ \\
C11 & $0.0303(10)$ & $0.0309(10)$ & $0.0317(10)$ & $0.0100(8)$ & $0.0074(8)$ & $0.0162(8)$ \\
C12 & $0.0713(17)$ & $0.0125(9)$ & $0.0354(11)$ & $0.0019(9)$ & $0.0030(11)$ & $0.0015(8)$ \\
C13 & $0.0272(9)$ & $0.0194(8)$ & $0.0287(9)$ & $-0.0055(7)$ & $0.0053(7)$ & $0.0001(7)$ \\
C14 & $0.0174(8)$ & $0.0239(9)$ & $0.0272(9)$ & $0.0060(7)$ & $0.0103(7)$ & $0.0119(7)$ \\
C15 & $0.0367(11)$ & $0.0482(12)$ & $0.0249(9)$ & $0.0116(10)$ & $0.0192(8)$ & $0.0138(9)$ \\
C16 & $0.0174(8)$ & $0.0298(11)$ & $0.0587(13)$ & $-0.0018(8)$ & $0.0084(8)$ & $0.0144(10)$ \\
C17 & $0.0203(8)$ & $0.0149(8)$ & $0.0139(7)$ & $0.0044(6)$ & $0.0025(6)$ & $0.0001(6)$ \\
C18 & $0.0363(11)$ & $0.0171(9)$ & $0.0273(10)$ & $-0.0016(8)$ & $-0.0037(8)$ & $0.0071(7)$ \\
& & & & & &
\end{tabular}




\begin{tabular}{lllllll} 
C6 & $0.048(3)$ & $0.0312(18)$ & $0.0220(14)$ & $0.0010(19)$ & $0.0181(16)$ & $0.0045(13)$ \\
C6A & $0.014(5)$ & $0.016(5)$ & $0.014(4)$ & $-0.010(4)$ & $0.004(4)$ & $0.001(4)$ \\
O1 & $0.042(5)$ & $0.033(3)$ & $0.051(4)$ & $0.010(2)$ & $0.026(3)$ & $0.028(2)$ \\
O1A & $0.033(5)$ & $0.095(17)$ & $0.048(8)$ & $0.014(7)$ & $0.012(5)$ & $0.055(9)$ \\
\hline
\end{tabular}

Geometric parameters $\left(A,{ }^{\circ}\right)$

\begin{tabular}{|c|c|c|c|}
\hline $\mathrm{O} 2-\mathrm{C} 17$ & $1.360(2)$ & C9-H9A & 0.9900 \\
\hline $\mathrm{O} 2-\mathrm{C} 2$ & $1.4071(19)$ & C9-H9B & 0.9900 \\
\hline $\mathrm{O} 3-\mathrm{C} 17$ & $1.200(2)$ & $\mathrm{C} 10-\mathrm{C} 10 \mathrm{~A}$ & $1.519(2)$ \\
\hline $\mathrm{C} 1-\mathrm{C} 2$ & $1.390(2)$ & $\mathrm{C} 10-\mathrm{H} 10 \mathrm{~A}$ & 0.9900 \\
\hline $\mathrm{C} 1-\mathrm{C} 10 \mathrm{~A}$ & $1.421(2)$ & $\mathrm{C} 10-\mathrm{H} 10 \mathrm{~B}$ & 0.9900 \\
\hline $\mathrm{C} 1-\mathrm{C} 14$ & $1.526(2)$ & $\mathrm{C} 11-\mathrm{H} 11 \mathrm{~A}$ & 0.9800 \\
\hline $\mathrm{C} 2-\mathrm{C} 3$ & $1.378(2)$ & C11-H11B & 0.9800 \\
\hline $\mathrm{C} 3-\mathrm{C} 4$ & $1.379(2)$ & $\mathrm{C} 11-\mathrm{H} 11 \mathrm{C}$ & 0.9800 \\
\hline $\mathrm{C} 3-\mathrm{H} 3$ & 0.9500 & $\mathrm{C} 12-\mathrm{H} 12 \mathrm{~A}$ & 0.9800 \\
\hline $\mathrm{C} 4-\mathrm{C} 4 \mathrm{~A}$ & $1.396(2)$ & $\mathrm{C} 12-\mathrm{H} 12 \mathrm{~B}$ & 0.9800 \\
\hline $\mathrm{C} 4-\mathrm{H} 4$ & 0.9500 & $\mathrm{C} 12-\mathrm{H} 12 \mathrm{C}$ & 0.9800 \\
\hline $\mathrm{C} 4 \mathrm{~A}-\mathrm{C} 10 \mathrm{~A}$ & $1.404(2)$ & $\mathrm{C} 13-\mathrm{H} 13 \mathrm{~A}$ & 0.9800 \\
\hline $\mathrm{C} 4 \mathrm{~A}-\mathrm{C} 4 \mathrm{~B}$ & $1.543(2)$ & $\mathrm{C} 13-\mathrm{H} 13 \mathrm{~B}$ & 0.9800 \\
\hline $\mathrm{C} 4 \mathrm{~B}-\mathrm{C} 5$ & $1.525(2)$ & $\mathrm{C} 13-\mathrm{H} 13 \mathrm{C}$ & 0.9800 \\
\hline $\mathrm{C} 4 \mathrm{~B}-\mathrm{C} 8 \mathrm{~A}$ & $1.545(2)$ & $\mathrm{C} 14-\mathrm{C} 15$ & $1.531(3)$ \\
\hline $\mathrm{C} 4 \mathrm{~B}-\mathrm{C} 13$ & $1.546(2)$ & $\mathrm{C} 14-\mathrm{C} 16$ & $1.537(3)$ \\
\hline $\mathrm{C} 5-\mathrm{C} 6$ & $1.352(3)$ & C14-H14 & 1.0000 \\
\hline $\mathrm{C} 5-\mathrm{C} 6 \mathrm{~A}$ & $1.565(11)$ & C15-H15A & 0.9800 \\
\hline $\mathrm{C} 5-\mathrm{H} 5$ & 0.9500 & C15-H15B & 0.9800 \\
\hline $\mathrm{C} 7-\mathrm{O} 1$ & $1.213(10)$ & $\mathrm{C} 15-\mathrm{H} 15 \mathrm{C}$ & 0.9800 \\
\hline $\mathrm{C} 7-\mathrm{O} 1 \mathrm{~A}$ & $1.215(16)$ & C16-H16A & 0.9800 \\
\hline $\mathrm{C} 7-\mathrm{C} 6$ & $1.453(4)$ & C16-H16B & 0.9800 \\
\hline $\mathrm{C} 7-\mathrm{C} 8$ & $1.526(3)$ & C16-H16C & 0.9800 \\
\hline $\mathrm{C} 7-\mathrm{C} 6 \mathrm{~A}$ & $1.557(9)$ & $\mathrm{C} 17-\mathrm{C} 18$ & $1.490(2)$ \\
\hline $\mathrm{C} 8-\mathrm{C} 11$ & $1.540(3)$ & C18-H18A & 0.9800 \\
\hline $\mathrm{C} 8-\mathrm{C} 12$ & $1.540(3)$ & C18-H18B & 0.9800 \\
\hline $\mathrm{C} 8-\mathrm{C} 8 \mathrm{~A}$ & $1.556(2)$ & $\mathrm{C} 18-\mathrm{H} 18 \mathrm{C}$ & 0.9800 \\
\hline $\mathrm{C} 8 \mathrm{~A}-\mathrm{C} 9$ & $1.530(2)$ & C6- 66 & 0.9500 \\
\hline $\mathrm{C} 8 \mathrm{~A}-\mathrm{H} 8 \mathrm{~A}$ & 1.0000 & C6A-H6A & 0.9500 \\
\hline $\mathrm{C} 9-\mathrm{C} 10$ & $1.522(2)$ & & \\
\hline $\mathrm{C} 17-\mathrm{O} 2-\mathrm{C} 2$ & $118.24(12)$ & $\mathrm{C} 4 \mathrm{~A}-\mathrm{C} 10 \mathrm{~A}-\mathrm{C} 1$ & $120.28(14)$ \\
\hline $\mathrm{C} 2-\mathrm{C} 1-\mathrm{C} 10 \mathrm{~A}$ & $117.54(14)$ & $\mathrm{C} 4 \mathrm{~A}-\mathrm{C} 10 \mathrm{~A}-\mathrm{C} 10$ & $121.20(14)$ \\
\hline $\mathrm{C} 2-\mathrm{C} 1-\mathrm{C} 14$ & $121.45(14)$ & $\mathrm{C} 1-\mathrm{C} 10 \mathrm{~A}-\mathrm{C} 10$ & $118.53(14)$ \\
\hline $\mathrm{C} 10 \mathrm{~A}-\mathrm{C} 1-\mathrm{C} 14$ & $120.98(14)$ & $\mathrm{C} 8-\mathrm{C} 11-\mathrm{H} 11 \mathrm{~A}$ & 109.5 \\
\hline $\mathrm{C} 3-\mathrm{C} 2-\mathrm{C} 1$ & $122.65(15)$ & $\mathrm{C} 8-\mathrm{C} 11-\mathrm{H} 11 \mathrm{~B}$ & 109.5 \\
\hline $\mathrm{C} 3-\mathrm{C} 2-\mathrm{O} 2$ & $118.38(14)$ & $\mathrm{H} 11 \mathrm{~A}-\mathrm{C} 11-\mathrm{H} 11 \mathrm{~B}$ & 109.5 \\
\hline $\mathrm{C} 1-\mathrm{C} 2-\mathrm{O} 2$ & $118.92(14)$ & $\mathrm{C} 8-\mathrm{C} 11-\mathrm{H} 11 \mathrm{C}$ & 109.5 \\
\hline $\mathrm{C} 2-\mathrm{C} 3-\mathrm{C} 4$ & $119.16(15)$ & $\mathrm{H} 11 \mathrm{~A}-\mathrm{C} 11-\mathrm{H} 11 \mathrm{C}$ & 109.5 \\
\hline $\mathrm{C} 2-\mathrm{C} 3-\mathrm{H} 3$ & 120.4 & $\mathrm{H} 11 \mathrm{~B}-\mathrm{C} 11-\mathrm{H} 11 \mathrm{C}$ & 109.5 \\
\hline $\mathrm{C} 4-\mathrm{C} 3-\mathrm{H} 3$ & 120.4 & $\mathrm{C} 8-\mathrm{C} 12-\mathrm{H} 12 \mathrm{~A}$ & 109.5 \\
\hline
\end{tabular}




\begin{tabular}{|c|c|}
\hline $\mathrm{C} 3-\mathrm{C} 4-\mathrm{C} 4 \mathrm{~A}$ & $121.16(15)$ \\
\hline $\mathrm{C} 3-\mathrm{C} 4-\mathrm{H} 4$ & 119.4 \\
\hline $\mathrm{C} 4 \mathrm{~A}-\mathrm{C} 4-\mathrm{H} 4$ & 119.4 \\
\hline $\mathrm{C} 4-\mathrm{C} 4 \mathrm{~A}-\mathrm{C} 10 \mathrm{~A}$ & $119.13(14)$ \\
\hline $\mathrm{C} 4-\mathrm{C} 4 \mathrm{~A}-\mathrm{C} 4 \mathrm{~B}$ & $118.45(14)$ \\
\hline $\mathrm{C} 10 \mathrm{~A}-\mathrm{C} 4 \mathrm{~A}-\mathrm{C} 4 \mathrm{~B}$ & $122.38(14)$ \\
\hline $\mathrm{C} 5-\mathrm{C} 4 \mathrm{~B}-\mathrm{C} 4 \mathrm{~A}$ & $111.34(13)$ \\
\hline $\mathrm{C} 5-\mathrm{C} 4 \mathrm{~B}-\mathrm{C} 8 \mathrm{~A}$ & $107.50(13)$ \\
\hline $\mathrm{C} 4 \mathrm{~A}-\mathrm{C} 4 \mathrm{~B}-\mathrm{C} 8 \mathrm{~A}$ & $108.47(13)$ \\
\hline $\mathrm{C} 5-\mathrm{C} 4 \mathrm{~B}-\mathrm{C} 13$ & $106.98(14)$ \\
\hline $\mathrm{C} 4 \mathrm{~A}-\mathrm{C} 4 \mathrm{~B}-\mathrm{C} 13$ & $107.10(13)$ \\
\hline $\mathrm{C} 8 \mathrm{~A}-\mathrm{C} 4 \mathrm{~B}-\mathrm{C} 13$ & $115.50(14)$ \\
\hline $\mathrm{C} 6-\mathrm{C} 5-\mathrm{C} 4 \mathrm{~B}$ & $120.85(18)$ \\
\hline $\mathrm{C} 4 \mathrm{~B}-\mathrm{C} 5-\mathrm{C} 6 \mathrm{~A}$ & $111.8(4)$ \\
\hline $\mathrm{C} 6-\mathrm{C} 5-\mathrm{H} 5$ & 119.6 \\
\hline $\mathrm{C} 4 \mathrm{~B}-\mathrm{C} 5-\mathrm{H} 5$ & 119.6 \\
\hline $\mathrm{O} 1-\mathrm{C} 7-\mathrm{C} 8$ & $118.7(5)$ \\
\hline $\mathrm{O} 1 \mathrm{~A}-\mathrm{C} 7-\mathrm{C} 8$ & $123.5(13)$ \\
\hline $\mathrm{C} 6-\mathrm{C} 7-\mathrm{C} 8$ & $118.54(17)$ \\
\hline $\mathrm{C} 8-\mathrm{C} 7-\mathrm{C} 6 \mathrm{~A}$ & $120.1(3)$ \\
\hline $\mathrm{C} 7-\mathrm{C} 8-\mathrm{C} 11$ & $106.34(15)$ \\
\hline $\mathrm{C} 7-\mathrm{C} 8-\mathrm{C} 12$ & $108.06(17)$ \\
\hline $\mathrm{C} 11-\mathrm{C} 8-\mathrm{C} 12$ & $108.06(18)$ \\
\hline $\mathrm{C} 7-\mathrm{C} 8-\mathrm{C} 8 \mathrm{~A}$ & $111.32(15)$ \\
\hline $\mathrm{C} 11-\mathrm{C} 8-\mathrm{C} 8 \mathrm{~A}$ & $108.33(15)$ \\
\hline $\mathrm{C} 12-\mathrm{C} 8-\mathrm{C} 8 \mathrm{~A}$ & $114.38(14)$ \\
\hline $\mathrm{C} 9-\mathrm{C} 8 \mathrm{~A}-\mathrm{C} 4 \mathrm{~B}$ & $109.20(13)$ \\
\hline $\mathrm{C} 9-\mathrm{C} 8 \mathrm{~A}-\mathrm{C} 8$ & $114.04(14)$ \\
\hline $\mathrm{C} 4 \mathrm{~B}-\mathrm{C} 8 \mathrm{~A}-\mathrm{C} 8$ & $116.83(14)$ \\
\hline $\mathrm{C} 9-\mathrm{C} 8 \mathrm{~A}-\mathrm{H} 8 \mathrm{~A}$ & 105.2 \\
\hline $\mathrm{C} 4 \mathrm{~B}-\mathrm{C} 8 \mathrm{~A}-\mathrm{H} 8 \mathrm{~A}$ & 105.2 \\
\hline $\mathrm{C} 8-\mathrm{C} 8 \mathrm{~A}-\mathrm{H} 8 \mathrm{~A}$ & 105.2 \\
\hline $\mathrm{C} 10-\mathrm{C} 9-\mathrm{C} 8 \mathrm{~A}$ & $109.53(14)$ \\
\hline $\mathrm{C} 10-\mathrm{C} 9-\mathrm{H} 9 \mathrm{~A}$ & 109.8 \\
\hline $\mathrm{C} 8 \mathrm{~A}-\mathrm{C} 9-\mathrm{H} 9 \mathrm{~A}$ & 109.8 \\
\hline $\mathrm{C} 10-\mathrm{C} 9-\mathrm{H} 9 \mathrm{~B}$ & 109.8 \\
\hline $\mathrm{C} 8 \mathrm{~A}-\mathrm{C} 9-\mathrm{H} 9 \mathrm{~B}$ & 109.8 \\
\hline $\mathrm{H} 9 \mathrm{~A}-\mathrm{C} 9-\mathrm{H} 9 \mathrm{~B}$ & 108.2 \\
\hline $\mathrm{C} 10 \mathrm{~A}-\mathrm{C} 10-\mathrm{C} 9$ & 114.07 (14) \\
\hline $\mathrm{C} 10 \mathrm{~A}-\mathrm{C} 10-\mathrm{H} 10 \mathrm{~A}$ & 108.7 \\
\hline $\mathrm{C} 9-\mathrm{C} 10-\mathrm{H} 10 \mathrm{~A}$ & 108.7 \\
\hline $\mathrm{C} 10 \mathrm{~A}-\mathrm{C} 10-\mathrm{H} 10 \mathrm{~B}$ & 108.7 \\
\hline $\mathrm{C} 9-\mathrm{C} 10-\mathrm{H} 10 \mathrm{~B}$ & 108.7 \\
\hline $\mathrm{H} 10 \mathrm{~A}-\mathrm{C} 10-\mathrm{H} 10 \mathrm{~B}$ & 107.6 \\
\hline
\end{tabular}

\begin{tabular}{|c|c|}
\hline $\mathrm{C} 8-\mathrm{C} 12-\mathrm{H} 12 \mathrm{~B}$ & 109.5 \\
\hline $\mathrm{H} 12 \mathrm{~A}-\mathrm{C} 12-\mathrm{H} 12 \mathrm{~B}$ & 109.5 \\
\hline $\mathrm{C} 8-\mathrm{C} 12-\mathrm{H} 12 \mathrm{C}$ & 109.5 \\
\hline $\mathrm{H} 12 \mathrm{~A}-\mathrm{C} 12-\mathrm{H} 12 \mathrm{C}$ & 109.5 \\
\hline $\mathrm{H} 12 \mathrm{~B}-\mathrm{C} 12-\mathrm{H} 12 \mathrm{C}$ & 109.5 \\
\hline $\mathrm{C} 4 \mathrm{~B}-\mathrm{C} 13-\mathrm{H} 13 \mathrm{~A}$ & 109.5 \\
\hline $\mathrm{C} 4 \mathrm{~B}-\mathrm{C} 13-\mathrm{H} 13 \mathrm{~B}$ & 109.5 \\
\hline $\mathrm{H} 13 \mathrm{~A}-\mathrm{C} 13-\mathrm{H} 13 \mathrm{~B}$ & 109.5 \\
\hline $\mathrm{C} 4 \mathrm{~B}-\mathrm{C} 13-\mathrm{H} 13 \mathrm{C}$ & 109.5 \\
\hline $\mathrm{H} 13 \mathrm{~A}-\mathrm{C} 13-\mathrm{H} 13 \mathrm{C}$ & 109.5 \\
\hline $\mathrm{H} 13 \mathrm{~B}-\mathrm{C} 13-\mathrm{H} 13 \mathrm{C}$ & 109.5 \\
\hline $\mathrm{C} 1-\mathrm{C} 14-\mathrm{C} 15$ & $111.07(15)$ \\
\hline $\mathrm{C} 1-\mathrm{C} 14-\mathrm{C} 16$ & $114.48(16)$ \\
\hline $\mathrm{C} 15-\mathrm{C} 14-\mathrm{C} 16$ & $111.19(17)$ \\
\hline $\mathrm{C} 1-\mathrm{C} 14-\mathrm{H} 14$ & 106.5 \\
\hline $\mathrm{C} 15-\mathrm{C} 14-\mathrm{H} 14$ & 106.5 \\
\hline $\mathrm{C} 16-\mathrm{C} 14-\mathrm{H} 14$ & 106.5 \\
\hline $\mathrm{C} 14-\mathrm{C} 15-\mathrm{H} 15 \mathrm{~A}$ & 109.5 \\
\hline $\mathrm{C} 14-\mathrm{C} 15-\mathrm{H} 15 \mathrm{~B}$ & 109.5 \\
\hline $\mathrm{H} 15 \mathrm{~A}-\mathrm{C} 15-\mathrm{H} 15 \mathrm{~B}$ & 109.5 \\
\hline $\mathrm{C} 14-\mathrm{C} 15-\mathrm{H} 15 \mathrm{C}$ & 109.5 \\
\hline $\mathrm{H} 15 \mathrm{~A}-\mathrm{C} 15-\mathrm{H} 15 \mathrm{C}$ & 109.5 \\
\hline $\mathrm{H} 15 \mathrm{~B}-\mathrm{C} 15-\mathrm{H} 15 \mathrm{C}$ & 109.5 \\
\hline $\mathrm{C} 14-\mathrm{C} 16-\mathrm{H} 16 \mathrm{~A}$ & 109.5 \\
\hline $\mathrm{C} 14-\mathrm{C} 16-\mathrm{H} 16 \mathrm{~B}$ & 109.5 \\
\hline $\mathrm{H} 16 \mathrm{~A}-\mathrm{C} 16-\mathrm{H} 16 \mathrm{~B}$ & 109.5 \\
\hline $\mathrm{C} 14-\mathrm{C} 16-\mathrm{H} 16 \mathrm{C}$ & 109.5 \\
\hline $\mathrm{H} 16 \mathrm{~A}-\mathrm{C} 16-\mathrm{H} 16 \mathrm{C}$ & 109.5 \\
\hline $\mathrm{H} 16 \mathrm{~B}-\mathrm{C} 16-\mathrm{H} 16 \mathrm{C}$ & 109.5 \\
\hline $\mathrm{O} 3-\mathrm{C} 17-\mathrm{O} 2$ & $123.70(15)$ \\
\hline $\mathrm{O} 3-\mathrm{C} 17-\mathrm{C} 18$ & $126.10(15)$ \\
\hline $\mathrm{O} 2-\mathrm{C} 17-\mathrm{C} 18$ & $110.19(14)$ \\
\hline $\mathrm{C} 17-\mathrm{C} 18-\mathrm{H} 18 \mathrm{~A}$ & 109.5 \\
\hline $\mathrm{C} 17-\mathrm{C} 18-\mathrm{H} 18 \mathrm{~B}$ & 109.5 \\
\hline $\mathrm{H} 18 \mathrm{~A}-\mathrm{C} 18-\mathrm{H} 18 \mathrm{~B}$ & 109.5 \\
\hline $\mathrm{C} 17-\mathrm{C} 18-\mathrm{H} 18 \mathrm{C}$ & 109.5 \\
\hline $\mathrm{H} 18 \mathrm{~A}-\mathrm{C} 18-\mathrm{H} 18 \mathrm{C}$ & 109.5 \\
\hline $\mathrm{H} 18 \mathrm{~B}-\mathrm{C} 18-\mathrm{H} 18 \mathrm{C}$ & 109.5 \\
\hline $\mathrm{C} 5-\mathrm{C} 6-\mathrm{C} 7$ & $124.3(2)$ \\
\hline $\mathrm{C} 5-\mathrm{C} 6-\mathrm{H} 6$ & 117.9 \\
\hline $\mathrm{C} 7-\mathrm{C} 6-\mathrm{H} 6$ & 117.9 \\
\hline $\mathrm{C} 7-\mathrm{C} 6 \mathrm{~A}-\mathrm{C} 5$ & $105.2(7)$ \\
\hline $\mathrm{C} 7-\mathrm{C} 6 \mathrm{~A}-\mathrm{H} 6 \mathrm{~A}$ & 127.4 \\
\hline $\mathrm{C} 5-\mathrm{C} 6 \mathrm{~A}-\mathrm{H} 6 \mathrm{~A}$ & 127.4 \\
\hline
\end{tabular}


Hydrogen-bond geometry $\left(A,{ }^{\circ}\right)$

$\mathrm{Cg} 1$ is the centroid of the benzene ring.

\begin{tabular}{lllll}
\hline$D-\mathrm{H} \cdots A$ & $D-\mathrm{H}$ & $\mathrm{H} \cdots A$ & $D \cdots A$ & $D-\mathrm{H} \cdots A$ \\
\hline $\mathrm{C} 4-\mathrm{H} 4 \cdots \mathrm{O} 1^{\mathrm{i}}$ & 0.95 & 2.50 & $3.411(10)$ & 159 \\
$\mathrm{C} 4-\mathrm{H} 4 \cdots \mathrm{O} 1 A^{\mathrm{i}}$ & 0.95 & 2.55 & $3.428(17)$ & 154 \\
$\mathrm{C} 10-\mathrm{H} 10 B \cdots \mathrm{O} 1 A^{\mathrm{ii}}$ & 0.99 & 2.55 & $3.328(17)$ & 136 \\
$\mathrm{C} 13-\mathrm{H} 13 C \cdots \mathrm{O} 3^{\mathrm{iii}}$ & 0.98 & 2.59 & $3.530(2)$ & 161 \\
$\mathrm{C} 18-\mathrm{H} 18 A \cdots C g 1^{\mathrm{i}}$ & 0.98 & 2.55 & $3.504(2)$ & 165
\end{tabular}

Symmetry codes: (i) $-x+1, y-1 / 2,-z$; (ii) $-x, y-1 / 2,-z$; (iii) $-x+1, y+1 / 2,-z+1$; (iv) $-x+1, y-1 / 2,-z+1$. 